\title{
Direct Measurement of Electrical Transport Through G-Quadruplex DNA with Mechanically Controllable Break Junction Electrodes**
}

\author{
Shou-Peng Liu, Samuel H. Weisbrod, Zhuo Tang, Andreas Marx, Elke Scheer, and Artur Erbe*
}

The need for miniaturization of devices for future nanoelectronic applications has led to the search for new constituents in molecular electronics. DNA is particularly interesting for applications in nanoelectronics circuits owing to its inherent properties, such as the predictable size and selfassembly of the stacked nucleobase pairs. ${ }^{[1,2]}$ In recent years, charge transport in double-stranded DNA (dsDNA) has attracted considerable attention because of its potential use in building blocks for future nanoelectronic circuits. The onedimensional nanowire conformation of DNA and its unique self-assembly ability ${ }^{[1,2]}$ can also be used in biochemical sensors. ${ }^{[3]}$ Theoretical studies suggested rather high conductance of DNA in the case of optimal and undisturbed overlap of the electronic orbitals of the $\pi$ electrons. ${ }^{[2]}$ Previously, several experimental groups reported high conductance of particular DNA molecules, ${ }^{[4-6]}$ whereas other experiments showed predominantly that the conductance of DNA was very low ${ }^{[7-10]}$ which is presumably due to variation in the contact geometries ${ }^{[11,12]}$ and its variable sequence and flexible conformation. However, it was recently reported that short dsDNA with a G-C sequence is more conductive than that with a A-T sequence. ${ }^{[13-15]}$ Certain guanine-rich DNA sequences, such as those found in telomeres at the end of chromosomes, can form stable four-stranded structures, which result from the stacking of several G-quartets folding into quadruplex structures. ${ }^{[16-18]}$ Furthermore, potential G-quartet-forming sequences have been found to be enriched in promoters of proto-oncogenes. ${ }^{[19]}$

Herein we present direct transport measurements on a G-quadruplex covalently wired between two gold electrodes realized by the mechanically controllable break junction (MCBJ) technique. The G-quadruplex shows a rather high

[*] S.-P. Liu, Prof. Dr. E. Scheer, Dr. A. Erbe ${ }^{[+]}$ Department of Physics, University of Konstanz Universitätsstrasse 10, 78464 Konstanz (Germany) Fax: (+49) 753-188-3091

E-mail: Artur.Erbe@uni-konstanz.de

S. H. Weisbrod, Dr. Z. Tang, Prof. Dr. A. Marx Department of Chemistry, University of Konstanz Universitätsstrasse 10, 78464 Konstanz (Germany)

${ }^{+}{ }^{+}$C Current address: Forschungszentrum Dresden-Rossendorf Bautzner Landstrasse 400, 01328 Dresden (Germany)

[**] We are grateful for discussions with J. C. Cuevas, M. Hettler, G. Cuniberti, M. Elstner, and J. Hartig. We thank B. Bornemann, H. Li, and T. Böhler for their contributions to this work. We gratefully acknowledge financial support from the Alfried Krupp von Bohlen und Halbach foundation, the DFG through SFB513 and SPP1243, and the Landesstiftung Baden-Württemberg.

D) Supporting information for this article is available on the WWW under http://dx.doi.org/10.1002/anie.201000022.

conductance. Interestingly, when the distance of both electrodes was reversibly varied over a several-nanometer span, this conductance behavior persists reproducibly. These unprecedented properties make G-quadruplexes interesting candidates for nanoelectronic applications in which varied distances between electrodes need bridging without loss of conductance.

Apart from the usual helical double-stranded form, DNA with particular sequences can also form stable four-stranded structures with repeated guanine bases. These structures result from the stacking of several G-quartets (planes of four guanines held together by eight hydrogen bonds; Figure 1 a). The G-quartet stacking can be further stabilized by cations (typically $\mathrm{K}^{+}$or $\mathrm{Na}^{+}$) located between two quartets. From various sequences, both intramolecular and intermolecular G-quadruplexes or G-wires with lengths of up to micrometers can be formed. For the human telomeric sequence that was used in our experiments, different structures are reported that containing either parallel, ${ }^{[20]}$ antiparallel, ${ }^{[21]}$ or even mixed parallel-antiparallel folding ${ }^{[22]}$ of the strands.

a)

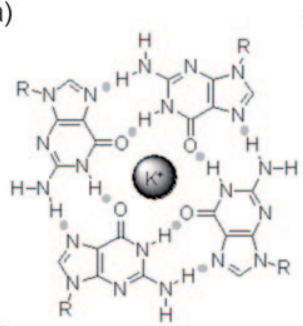

c)

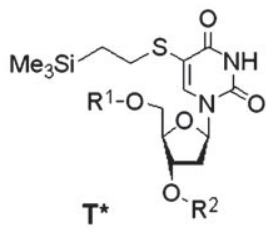

d)

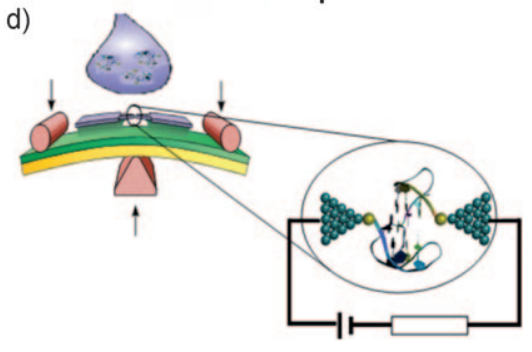

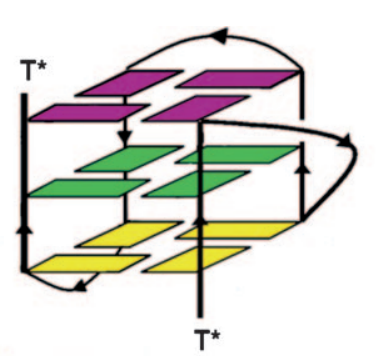

Figure 1. Structure of the oligonucleotides. a) G-quartet plane with a cation in the center (gray sphere). Gray spots: hydrogen bonds. b) A G-quadruplex structure with mixed parallel-antiparallel folding based on a structure in solution derived with NMR spectroscopy. ${ }^{[22]}$ Black lines show the backbone orientation. Colored squares show the three stacked quartet planes. c) The molecule terminates with modified thymine bases $\left(T^{*}\right)$ to allow binding to gold electrodes. d) MCB setup A suspended narrow (100 $\mathrm{nm}$ wide) metallic bridge is produced on a flexible substrate. The substrate is bent by pushing the central support with respect to the two outer supports. This bending of the substrate causes the metallic bridge to break, producing two closely spaced, one-atom-sharp electrodes. The DNA is deposited by placing a droplet of DNA solution on top of the sample. 
Although no structure in a vacuum has been reported to date, there is evidence that G-quadruplexes with stacked quartet planes do exist in the gas phase. ${ }^{[23,24]}$ Because of the higher number of overlapping $\pi$ electrons between quartet planes compared to dsDNA and owing to its less-flexible structure, polarizability measurements have led to the prediction that DNA in the long G-quadruplex structure may have higher conductance than dsDNA. ${ }^{[25]}$ Furthermore, $\mathrm{K}^{+}$or $\mathrm{Na}^{+}$ions trapped in the quadruplex center may also improve the conductance because they provide hybridization with the base stack and an intrinsic doping factor, similar to electronic modifications introduced in dsDNA helices by metal cations inserted in the inner core. ${ }^{[26-29]}$ To measure the charge transport through a G-quadruplex, we used a mechanically controlled break junction (MCBJ) experiment (see Figure 1d and the Supporting Information) in which a single oligonucleotide that can form a G-quadruplex with three stacked quartet planes (Figure 1 b,c) bridges the electrodes. The G-quadruplex was modified in a way that allows direct coupling of the nucleobase $\pi$ system on the top and bottom part of the quadruplex to the gold electrode of the MCBJ by a thiol-gold interaction. ${ }^{[30]}$

First, we measured the dependence of charge transfer on the distance between the electrodes bridged by oligonucleotide G1 $\left(5^{\prime}-\left(\mathrm{T}^{*} \mathrm{G}_{3}[\mathrm{TTAGGG}]_{3} \mathrm{~T}^{*}\right)-3^{\prime}\right),{ }^{[30]}$ which is known to form stable G-quadruplexes. $\mathrm{T}^{*}$ is a modified thymidine residue that induces coupling of the molecules to the metal electrodes by the sulfur atoms (Figure 1c). Figure $2 \mathrm{a}-\mathrm{c}$ show examples of measured resistance upon shortening and lengthening the distance for two samples of G1. For sample 1 (Figure 2a), a pronounced plateau in resistance as a function of distance was observed both when opening and closing the gap of the electrodes. The length of the plateau is in the order of $2 \mathrm{~nm}$. The resistance value in the plateau region fluctuates over a range of approximately $10^{8} \Omega$. This behavior is reproducible for about 30 repetitions until eventually the plateau vanishes (green and blue curve). The latter fact signals the loss of the molecule in the junction as supported by a change of the current-voltage $(I-V)$ characteristics. A similar behavior is found when the experiments were repeated with sample 2 (Figure $2 b, c$ ), with the minor difference that the plateaus are more pronounced upon opening the electrodes. Again the traces are stable for several repetitions. The length of the plateau varies from $0.5 \mathrm{~nm}$ to $3.5 \mathrm{~nm}$. In stark difference to equivalent investigations on dsDNA in which plateaus as a function of distance have also been observed, ${ }^{[31]}$ the step series is reproducible in many details: the steps occur at the same distances and the same resistance values. For other molecules as well as atomic contacts, the opening traces vary from opening to opening, and typical resistance or distance values can be deduced only statistically. ${ }^{[15,32]}$ The repeatable opening and closing cycles are observed when the electrodes are opened by at most $4 \mathrm{~nm}$ up to a resistance of typically more than $10^{10} \Omega$. If the distance is increased further, the steplike progress changes or disappears completely. This complete break is associated with a breaking of a gold-gold bond in the electrodes ${ }^{[33]}$ and it therefore distorts the local geometry in total.

We interpret the plateaus as a signature of the unfolding and folding process of the molecules in the junction. In the contact geometry, the stretching force is applied perpendicular to the G4 planes, which makes them slide with respect to each other, giving rise to the fluctuations of the resistance on a plateau. Recent measurements of bonding strengths in G-quadruplex structures reveal forces of between $25 \mathrm{pN}$ and $50 \mathrm{pN},{ }^{[34,35]}$ which are orders of magnitude below estimated values for the typical thiol-gold bond $(1.5 \mathrm{nN})$ and bonds within the metal $(1 \mathrm{nN}) .^{[35]}$ The plateau resistance remains almost constant while the G-quadruplex conformation is present. The slightly different behavior between sample 1 and sample 2 can be attributed to the different positions on the electrodes at which the G-quadruplex binds.

The interpretation of the opening and closing curves is supported by the current-voltage $(I-V)$ characteristics mea- 
sured in the different stretching states (Figure 3). As an indication for the stretching state, we measured the resistance $R$ of the junction during the opening and closing curves measured at a bias voltage of $V_{\mathrm{sd}}=100 \mathrm{mV}$. Figure $3 \mathrm{~d}$ indicates the three regions in which the $I-V$ curves in Figure $3 \mathrm{a}-\mathrm{c}$ were measured. For small resistances $R<10^{6} \Omega$, the $I-V$ curves are mainly linear in a voltage range up to approximately $1 \mathrm{~V}$; we attribute this effect to electronic

several occasions we found a hysteretic behavior with a systematically higher current when decreasing the absolute value of the voltage. These asymmetries and hystereses hamper a quantitative description of the shape. However, the polarity of the asymmetries vary from junction to junction, indicating that the nonlinearities are in fact due to current transport through a single (or a small number of) molecules. ${ }^{[38]}$
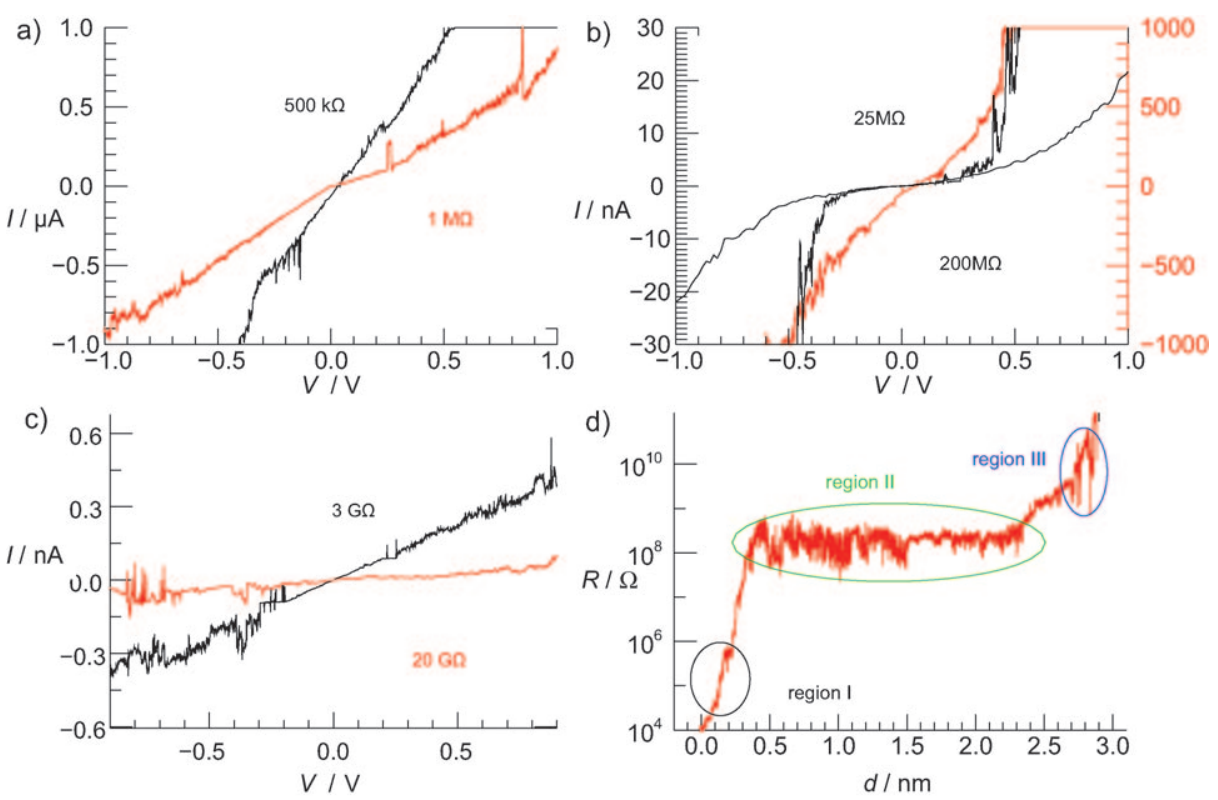

Figure 3. Current-voltage characteristics. a-c) Typical $I-V$ curves recorded on samples 1 and 2 at different stages of defolding (d). a) In region I, b) in region II (on the plateau), and c) in region III. d) Resistance versus distance of sample 1 with regions I, II, and III indicated.

tunneling across a barrier. ${ }^{[36]}$ There is no indication of current transport through the molecular orbitals, because the current achieved by direct tunneling exceeds the current that can be driven through the molecule by an order of magnitude. In the plateau region, that is, for $10^{7} \Omega<R(V=100 \mathrm{mV})<10^{9} \Omega$, the $I-V$ curves are highly non-linear, with a typical $\mathrm{S}$ shape as found earlier for other DNA derivatives. ${ }^{[1,2]}$ However, the current level in our samples is larger than found in most of the reports about dsDNA ${ }^{[1]}$ and arrives at several hundred nanoamperes at a voltage of approximately $0.7 \mathrm{~V}$. We used a current limitation of $1 \mu \mathrm{A}$ in our set-up, because exceeding this value occasionally results in destruction of the contact. We note that standard tunneling or semiconductor transport models cannot successfully describe the $I-V$ curves, and usually find a suppression of the current at about $0 \mathrm{~V}$, which can arise if the charging energy of the electrons on the molecules is larger than the coupling between the electrodes and the molecules. ${ }^{[37]}$ The interplay of these energies depends on the molecular linkers to the metal and the geometry of the junction. In our measurements, the size of the voltage range with reduced conductance depends on the stretching length and the resistance. Because of the pronounced resistance fluctuations, a detailed correlation is hard to establish, but there is a tendency to find larger gaps at larger distances. In
When leaving the plateau region upon further stretching of the contact, the $I-V$ curves change back again to a merely linear behavior, which we again interpret as tunneling. In the overstretched situation, the conformation of the molecule is a single strand of DNA. Tunnel charge transport is the typical transport mechanism for single-stranded DNA. ${ }^{[1,15]}$

Finally, we also studied the transport measurements on the control oligonucleotide $\mathrm{C} 1$ (5'-d$\left(\mathrm{T}^{*} \mathrm{C}_{3}\left[\mathrm{TTACCC}_{3} \mathrm{~T}^{*}\right)-3^{\prime}\right)$ which does not form a quadruplex structure, ${ }^{[39]}$ and the formation of an imotif structure can only occur at acidic $\mathrm{pH}(\leq 5.9) .{ }^{[40]}$ No reproducible plateaus were seen in the opening and closing curves (see Figure $2 \mathrm{~d}$ ), and the $I-V$ curves were either unstable (jumping while sweeping the voltage) or roughly linear as expected for tunneling through a barrier. This behavior has been found experimentally for single-stranded DNA contacted by carbon nanotubes. ${ }^{[15]}$

In conclusion, we have demonstrated that G-quadruplex DNA is able to transport considerable currents at reasonable transport voltages in the range of $1 \mathrm{~V}$. Although the $I-V$ characteristics are nonlinear and the resistance thus strongly depends on the applied voltage, the resistance is quite independent of the elongation of the molecule. This feature is unique for molecular junctions, as their resistance usually strongly varies upon tiny changes of the contact configuration. Length-independent conductance has been found for atomic wires before $;^{[32]}$ however, the possible length variation is limited to only a few Ångstroms. G-quadruplexes may thus serve as flexible leads for connecting microscale electrodes to functional molecular units, such as molecular switches, which undergo a pronounced conformational change upon switching, for example with photochromic azobenzene derivatives. $^{[30]}$

\section{Experimental Section}

$5^{\prime}-\mathrm{T}^{*} \mathrm{GGG}$ [TTAGGG] $]_{3} \mathrm{~T}^{*}-3^{\prime}$ (G1) and $5^{\prime}$-T*CCC[TTACCC $]_{3} \mathrm{~T}^{*}-3^{\prime}$ (C1) were synthesized by standard automated DNA oligonucleotide synthesis. ${ }^{[25]}$ Mass spectrometric analysis verified the integrity of the 
oligonucleotides. For immobilization, the oligonucleotides were diluted with PBS buffer $\left(40 \mathrm{mmK}_{2} \mathrm{PO}_{4}\right.$ and $26 \mathrm{~mm} \quad \mathrm{KH}_{2} \mathrm{PO}_{4}$ $\mathrm{pH}$ 6.98) to a final concentration of $10 \mu \mathrm{mol} \mathrm{L}^{-1}$. In this solution (ca. $100 \mathrm{~mm} \mathrm{~K}^{+}$), the 22 mer G1 oligonucleotide would adapt a parallel or mixed parallel/antiparallel quadruplex structure (see Figure 1), whilst the $\mathrm{C} 1$ was still in single-stranded conformation, as shown by $\mathrm{CD}$ spectroscopy. ${ }^{[36,39]}$

Received: January 4, 2010

Published online: March 26, 2010

Keywords: DNA · break junctions - molecular electronics nanoelectronics

[1] D. Porath, G. Cuniberti, R. Di Felice, Top. Curr. Chem. 2004, 237, 183.

[2] R. G. Endres, D. L. Cox, R. R. P. Singh, Rev. Mod. Phys. 2004, 76, 195.

[3] K. J. Odenthal, J. J. Gooding, Analyst 2007, 132, 603.

[4] A. Y. Kasumov, M. Kociak, S. Gueron, B. Reulet, V. T. Volkov, D. V. Klinov, H. Bouchiat, Science 2001, 291, 280.

[5] A. Y. Kasumov, D. V. Klinov, P. E. Roche, S. Gueron, H. Bouchiat, Appl. Phys. Lett. 2004, 84, 1007.

[6] H.-W. Fink, C. Schönenberger, Nature 1999, 398, 407.

[7] Y. Zhang, R. H. Austin, J. Kraeft, E. C. Cox, N. P. Ong, Phys. Rev. Lett. 2002, 89, 198102.

[8] H. Cohen, C. Nogues, R. Naaman, D. Porath, Proc. Natl. Acad. Sci. USA 2005, 102, 11589.

[9] E. Braun, Y. Eichen, U. Sivan, G. Ben-Yoseph, Nature 1998, 391, 775 .

[10] D. Porath, A. Bezryadin, S. de Vries, and C. Dekker, Nature 2000, 403, 635 .

[11] H. Watanabe, C. Manabe, T. Shigematsu, K. Shimotani, M. Shimizu, Appl. Phys. Lett. 2001, 79, 2462.

[12] X. Guo, A. A. Gorodetsky, J. Hone, J. K. Barton, C. Nuckolls, Nat. Nanotechnol. 2008, 3, 163.

[13] K. H. Yoo, D. H. Ha, J. O. Lee, J. W. Park, J. Kim, J. J. Kim, H. Y. Lee, T. Kawai, H. Y. Choi, Phys. Rev. Lett. 2001, 86, 8719.

[14] B. Q. Xu, P. M. Zhang, X. L. Li, N. J. Tao, Nano Lett. 2004, 4, 1105.

[15] S. Roy, H. Vedala, A. D. Roy, D. H. Kim, M. Doud, K. Mathee, H. K. Shin, N. Shimamoto, V. Prasad, W. Choi, Nano Lett. 2008 , $8,26$.

[16] C. W. Greider, E. H. Blackburn, Cell 1985, 43, 405.

[17] N. W. Kim, M. A. Piatyszek, K. R. Prowse, C. B. Harley, M. D. West, P. L. Ho, G. M. Coviello, W. E. Wright, S. L. Weinrich, J. W. Shay, Science 1994, 266, 2011.
[18] J. T. Davis, G. P. Spada, Chem. Soc. Rev. 2007, 36, 296.

[19] J. L. Huppert, S. Balasubramanian, Nucleic Acids Res. 2005, 33, 2908.

[20] G. N. Parkinson, M. P. H. Lee, S. Neidle, Nature 2002, 417, 876.

[21] Y. Wang, D. J. Patel, Structure 1993, 1, 263.

[22] J. Dai, C. Punchihewa, A. Ambrus, D. Chen, R. A. Jones, D. Yang, Nucleic Acids Res. 2007, 35, 2440.

[23] M. Rueda, F. J. Luque, M. Orozco, J. Am. Chem. Soc. 2006, 128, 3608.

[24] V. Gabelica, F. Rosu, E. De Pauw, J. Lemaire, J.-C. Gillet, J.-C. Poully, F. Lecomte, G. Gregoire, J.-P. Schermann, C. Desfrancois, J. Am. Chem. Soc. 2008, 130, 1810.

[25] H. Cohen, T. Sapir, N. Borovok, T. Molotsky, R. Di Felice, A. B. Kotlyar, D. Porath, Nano Lett. 2007, 7, 981.

[26] K. Tanaka, A. Tengeiji, T. Kato, N. Toyama, M. Shionoya, Science 2003, 299, 1212.

[27] P. Aich, S. L. Labiuk, L. W. Tari, L. J. Delbaere, W. J. Roesler, K. J. Falk, R. P. Steer, J. S. Lee, J. Mol. Biol. 1999, 294, 477.

[28] A. Rakitin, P. Aich, C. Papadopoulos, Y. Kobzar, A. S. Vedeneev, J. S. Lee, J. M. Xu, Phys. Rev. Lett. 2001, 86, 3670.

[29] K. Tanaka, Y. Yamada, M. Shionoya, J. Am. Chem. Soc. 2002, 124,8802 .

[30] B. Bornemann, S. Liu, A. Erbe, E. Scheer, A. Marx, ChemPhysChem 2008, 9, 1241.

[31] N. Kang, A. Erbe, E. Scheer, New J. Phys. 2008, 10, 023030.

[32] N. Agraït, A. L. Yeyati, J. M. v. Ruitenbeek, Phys. Rep. 2003, 377, 81.

[33] J. Reichert, H. B. Weber, M. Mayor, H. von Löhneysen, Appl. Phys. Lett. 2003, 82, 4137.

[34] Z. Yu, J. D. Schonhoft, S. Dhakal, R. Bajracharya, R. Hegde, S. Basu, H. Mao, J. Am. Chem. Soc. 2009, 131, 1876.

[35] S. Lynch, H. Baker, S. G. Byker, D. Zhou, K. Sinniah, Chem. Eur. J. 2009, 15, 8113.

[36] W. Wang, T. Lee, M. A. Reed, Phys. Rev. B 2003, 68, 035416.

[37] F. Zahid, M. Paulsson, S. Datta, Handbook of Nanoscience, Engineering and Technology, CRC, Boca Raton, 2002.

[38] J. Reichert, R. Ochs, D. Beckmann, H. B. Weber, M. Mayor, H. von Löhneysen, Phys. Rev. Lett. 2002, 88, 176804.

[39] In principle, the quadruplex structure can be changed by the addition of ions to the buffer. However, this process changes the overall conductance of the junction, and therefore changes of the molecular conformation cannot be detected. The behavior that is described herein was not found for several different DNA sequences, which we characterized in an identical contacting process with a large number of repetitions.

[40] K. Guo, V. Gokhale, L. H. Hurley, D. Sun, Nucleic Acids Res. 2008, 36, 4598. 